\title{
Practical and Exact Synchronization of Complex Networks of Fractional Order Nonlinear Systems
}

\author{
Rafael Martínez-Martínez ${ }^{1,2}$, Armando Fabián Lugo-Peñaloza ${ }^{*}, 3,4$, Jorge A. León ${ }^{1}$ and \\ Guillermo Fernández-Anaya ${ }^{3}$
}

\author{
${ }^{1}$ Automatic Control Department, CINVESTAV-IPN, Av. IPN 2508, 07360 D.F., México \\ ${ }^{2}$ Systems Academy, UPIITA-IPN, Av. IPN 2580, 07340 D.F., México \\ ${ }^{3}$ Physics and Mathematics Department, Universidad Iberoamericana, Prol. Paseo de la Reforma 880,01219 \\ D.F., México \\ ${ }^{4}$ Mechatronics Academy, UPIITA-IPN, Av. IPN 2580, 07340 D.F., México
}

\begin{abstract}
Based on stability theorems for nonlinear differential systems with commensurate fractional orders and on the use of a nonlinear coupling that does not require the total elimination of the nonlinear part of the system, we have obtained an analytical result to ensure the practical and exact synchronization of complex networks with fractional nonlinear order systems as elements. As an application, we present the synchronization with different kinds of fractional differential systems. We use some numerical results to verify the effectiveness of the proposed method.
\end{abstract}

Keywords: Complex networks, fractional order systems, exact synchronization, practical synchronization.

\section{INTRODUCTION}

The interactions among different physical systems are common within the nature and artificial fields and they give rise to collective behaviours and in several works it has been pointed out that fractional differential operators are at least an alternative method to describe some physical systems; this is one of the reasons to study the synchronization of different kind of systems that are in interaction, forming complex networks, specially where such systems are mathematically described by means of fractional operators and therefore form complex fractional networks. Synchronization is a phenomenon that arises in many areas of science and technology. On the other hand, this kind of phenomena occurs all the time in nature e.g., fireflies flashing at the same time, crickets singing in synchrony, the heart cells that pump at the same beat, etc. $[1,2]$.

The synchronization of a dynamically coupled network (dynamic systems in interaction, known as complex networks) has been studied for many years, within a common structure with base on the nonlinear dynamics of the systems of the network. The interest on such networks is due to their frequent arising in natural and artificial systems.

The construction of new models of the complex dynamic networks [3-5] has led to a fascinating set of common problems concerning the way a network facilitates and limits

*Address correspondence to this author at the Physics and Mathematics Department, Universidad Iberoamericana, Prol. Paseo de la Reforma 880, 01219 D. F., México; Tel: +52 (55) 5950-4000/9177-4400;

E-mails: armando.lugo@correo.uia.mx, aflugop@gmail.com the collective behaviours. In particular, many efforts have been made in oscillator synchronization [6-8] and biological nervous networks synchronization $[9,10]$.

We study fractional complex networks using fractional calculus, which has a history almost as large as the one of the ordinary calculus, however its applications in physics and in the engineering are recently appearing $[11,12]$. There are many known systems where the modelling with fractional operators has turned out to be useful, for example systems involved in phenomena such as: visco-elasticity [13], dielectric polarization, electrode-electrolyte polarization, electromagnetic waves, chaotic dynamics and its synchronization [14-18]), stability [19-25], network synchronization [26], quantum evolution of complex systems [27], digital image processing, Euler-Lagrange equations, fractional kinetics, Polymer Physics, Biophysics, among others [12].

Namely, in encryption, the fractional order chaotic systems have more adjustable variables than integer order chaotic systems, therefore it is widely believed that fractional-order chaotic systems can be applied in encryption efficiently and thus enlarge the key space [28, 29].

On the other hand, during the last decade some extensions with fractional order operators for integer order chaotic systems have been investigated, some of these works are [30-32]. There are many different works on the synchronization of fractional complex systems [28, 33-35], and on the synchronization of fractional-order chaotic systems [36-40], in such works several schemes are proposed that ensure that the error dynamics satisfies [24] 
type conditions, this means that the error dynamics must hold a linear relation. In this work a synchronization scheme is proposed that asserts that the error dynamics satisfies [25] type conditions, which means that is not necessary to cancel the nonlinear part of the error dynamics, but it does imply a particular kind of synchronization, which is known as practical synchronization.

This work is ordered as follows: in Section 2 basic definitions of fractional order systems are presented, in Section 3 the definition of a complex network is introduced and the problems of practical and exact synchronization are studied, in Section 4 an application of fractional order differential systems is analyzed, and finally in section 5 we give some conclusions are given.

\section{PRELIMINARIES}

There are several definitions of a fractional derivative of order $\alpha \in \mathbb{R}^{+}[11,41,42]$. We will use the Caputo fractional operator in the definition of fractional order systems, because the meaning of the initial conditions for systems described using this operator is the same as for integer order systems.

Definition 1 (Caputo Fractional Derivative) The Caputo fractional derivative of order $\alpha \varepsilon \mathbb{R}^{+}$of a function $x$ is defined as (see [11]):

$x^{(\alpha)}={ }_{t_{0}} D_{t}^{\alpha} x=\frac{1}{\Gamma(m-\alpha)} \int_{t 0}^{t} \frac{d^{m} x(\tau)}{d \tau^{m}}(t-\tau)^{m-\alpha-1} d \tau$,

where: $m-1 \leq \alpha \leq m, \frac{d^{m} x(\tau)}{d \tau^{m}}$ is the $m$-th derivative of $x$ in the usual sense, $m \in \mathbb{N}$, and $\Gamma$ is the gamma function ${ }^{1}$.

Due to the problem that lies in the use of time translations in fractional operators, some researchers have claimed [43-47] that if one wants to solve the usual initial value problem is necessary to know the initial trajectory in addition to the initial condition. Our work is focused on systems where the initial trajectory is unknown, and for such systems this modelling tool is enough.

We consider the initial condition problem for an autonomous fractional order nonlinear system, with $0<\alpha<1$ :

$x^{(\alpha)}=f(x), \quad x(0)=x_{0}$,

where $x \in \Omega \subset \mathbb{R}^{n}, f: \Omega \rightarrow \mathbb{R}^{n}$ is a Lipschitz continuous function $^{2}$, with $x_{0} \in \Omega \subset \mathbb{R}^{n}$ and $\Omega$ is a region in $\mathbb{R}^{n}$.

For fractional order linear time invariant systems, i. e., $f(x)=A x, \mathrm{~A} \in \mathbb{R}^{n \times n}$ we can guarantee the stability in the Lyapunov sense. The following result [24] deals with the system

$x^{(\alpha)}=A x, \quad x(0)=x_{0}, 1,2$

${ }^{1}$ To simplify the notation we omitted the time dependence in $x^{(\alpha)}$, in what follows we take $t_{0}=0$.
Theorem 1 ([24]) Let $0<\alpha<1$. The system (3) is:

1. Asymptotically stable if and only if $|\arg (\operatorname{spec}(A))|$ $>\alpha \pi / 2, i=1,2, \cdots, n$, where $(\operatorname{spec}(A))$ denotes the argument of the eigenvalues of $A$. In this case, the component of the state decays towards 0 like $t^{-\alpha}$;

2. Stable if and only if either it is asymptotically stable, or those critical eigenvalues which satisfy $|\arg (\operatorname{spec}(A))|=\alpha \pi / 2$ have geometric multiplicity one.

For fractional order nonlinear systems we use the following result [25]:

Theorem 2 ([25]) Let us consider the n-dimensional fractional dynamic system

$x^{(\alpha)}=A x+f(x)$

where: $A \in \mathbb{R}^{n \times n}, f: \Omega \rightarrow \mathbb{R}^{n}$ is a nonlinear function of $x$ and $0<\alpha<1$. If:

1. The solution $x(t)=0$ of $x^{(\alpha)}=A x$ is asymptotically stable, and $\alpha \rho(A)>1$,

2. $f(0)=0$ and $\lim _{\|x\| \rightarrow 0} \frac{\|f(x)\|}{\|x\|}=0$,

then $x(t)=0$ for $0 \leq t_{0} \leq t$, is a stable solution of (4).

For an $n$-dimensional nonlinear fractional order dynamic system with the Caputo derivative,

$x^{(\alpha)}=A x+g(t, x), \quad t>t_{0}$,

under the initial conditions

$\left.x^{(\alpha-k)}(t)\right|_{t=t_{0}}=x_{k-1}, \quad k=1,2$,

where $x \in \mathbb{R}^{n}, A \in \mathbb{R}^{n \times n}, 1<\alpha<2, \mathrm{~g}(t, x):\left[t_{0}, \infty\right) \times \mathbb{R}^{n} \rightarrow \mathbb{R}^{n}$ is a continuous function which holds the Lipschitz conditions relative to $x$, and $g(t, 0)=0$.

Theorem 3 ([26]) Let us consider the system defined by (5) and (6), if the matrix $A$ is such that $|\arg (\operatorname{spec}(A))| \neq 0$, $|\arg (\operatorname{spec}(A))|>\alpha \pi / 2, \alpha+\left(\frac{1}{\|A\|}\right)<2$, and suppose that the function $g(t, x)$ satisfies uniformly

$\lim _{x \rightarrow \infty} \frac{\|g(t, x)\|}{\|x\|}=0, \quad t \in\left[t_{0}, \infty\right)$,

then $x(t)=0$ is an asymptotically stable solution of (5).

The proof of this theorem for the Caputo derivative follows from the proof of Theorem 3.3 in [49] and the

${ }^{2}$ This ensures the uniqueness of the solution [48]. 
application of Lemma 2.7 in [49] together with the Gronwall-Bellman inequality.

We make use of all these results to obtain the stabilization or asymptotic stabilization of the origin of the dynamical systems of synchronization error for every element in the complex fractional network, this is equivalent to obtain the practical or exact synchronization of the network. It is clear from these previous results that the practical synchronization can be obtained for systems with $0<\alpha<1$ and the exact synchronization for systems with $1<\alpha<2$.

\section{SYNCHRONIZATION OF FRACTIONAL COMPLEX NETWORKS}

The synchronization scheme that is presented in this Section is roughly illustrated in Fig. (1). The individual controls are dependent on the master system and on the network topology.

We will define a synchronization error for each of the elements of the complex fractional network, and then we will search for the stabilization around the origin of the fractional-order nonlinear dynamical system of the error in order to guarantee the synchronization of all the elements within the network.

We propose the general form of a complex fractional network. That is, this section is devoted to the study of $N$ systems with fractional order, which are interacting under a certain interconnection, see the Fig. 2a), of the form

$x_{i}^{(\alpha)}=A_{i} x_{i}+f_{i}\left(x_{i}\right)+\sum_{j=1}^{N} H_{i j}\left(x_{j}\right)$,

$x_{i}^{(\alpha)}=A_{i} x_{i}+\sum_{j=1}^{N} \mathcal{A}_{i j} x_{j}+\sum_{j=1}^{N} \mathcal{F}_{i j}\left(x_{j}\right)$,

where, for every $i=1,2, \cdots, N$ we have

1. $x_{i} \in \mathbb{R}^{n}$ is the state of the $i$-th slave system,

2. $A_{i}: \in \mathbb{R}^{n \times n}$ represents the linear part of the $i$-th slave system,

3. $f_{i} \in \mathbb{R}^{n}$ represents the nonlinear part of the $i$-th slave system,

4. $\quad H_{i j} \in \mathbb{R}^{n}$ describes how the $j$-th slave system is connected with the $i$-th slave system, i. e., this relation specifies the strength and the topology of the interconnection of the systems,

5. $\mathcal{A}_{l j} \in \mathbb{R}^{n \times n}$ represents the linear part of $H_{i j}$,
6. $\quad \mathcal{F}_{i j} \in \mathbb{R}^{n}$ is $f_{i}$ plus the nonlinear part of $H_{i j}$.

In general, not all the systems are interconnected in the same way, and we assume that $f_{i}$ and $H_{i j}$ are Lipschitz continuous functions.

Let us consider another fractional dynamical system with the same fractional order, which will be the master system:

$x_{\mathcal{M}}^{(\alpha)}=A_{\mathcal{M}} x_{\mathcal{M}}+f_{\mathcal{M}}\left(x_{\mathcal{M}}\right)$

where $x_{\mathcal{M}} \in \mathbb{R}^{n}$ is the state vector, $A_{\mathcal{M}} \in \mathbb{R}^{n \times n}$ and $f_{\mathcal{M}} \in \mathbb{R}^{n}$ is a Lipschitz continuous function.

Also let us consider a complex fractional network with elements of the form

$x_{i}^{(\alpha)}=A_{i} x_{i}+\sum_{j=1}^{N} \mathcal{A}_{i j} x_{j}+\sum_{j=1}^{N} \mathcal{F}_{i j}\left(x_{j}\right)+u_{i}$,

which will be the slave systems and $u_{i}$ is a new interconnection, with $x_{\mathcal{M}}$, that we want to choose in such a way that we synchronize the entire network with the master system.

In other words; in the master-slave synchronization scheme $x_{\mathcal{M}}$ describes the target signal, while $x_{i}$ represents the response signal, see Fig. 2b). Therefore the synchronization problem can be established as follows:

Given the master system (10) and our slave system (11), we should be determinate an interconnection $u_{i}$, such that the target signal is synchronized with the response signal. Therefore, if we define the synchronization error by

$e_{i}=x_{\mathcal{M}}-x_{i}$,

then we will need to find the conditions over $u_{i}$ such that $\lim _{t \rightarrow \infty}\left|e_{i}(t)\right|=0$, for every $i$.

\subsection{Practical Synchronization of Fractional Complex Networks}

With this master-slave scheme we will prove that practical synchronization [50] is achieved for $0<\alpha<1$.

Theorem 4 Consider a complex fractional network whose elements are of the form (11), where $\mathcal{A}_{j,} \in \mathbb{R}^{n \times n}$ and $\mathcal{F}_{i i}(0)=0 \quad$ and $\quad \lim _{x \rightarrow \infty} \frac{\left\|\mathcal{F}_{i i}\left(x_{i}\right)\right\|}{\left\|x_{i}\right\|}=0 \quad$ hold, $\quad$ for $i, j \in\{1,2, \cdots, N\}$. Moreover, assume that there are $B_{1}, B_{2}, \cdots, B_{n} \in \mathbb{R}^{n \times n} \quad$ such that $\quad A_{i}+B_{i} \quad$ satisfies

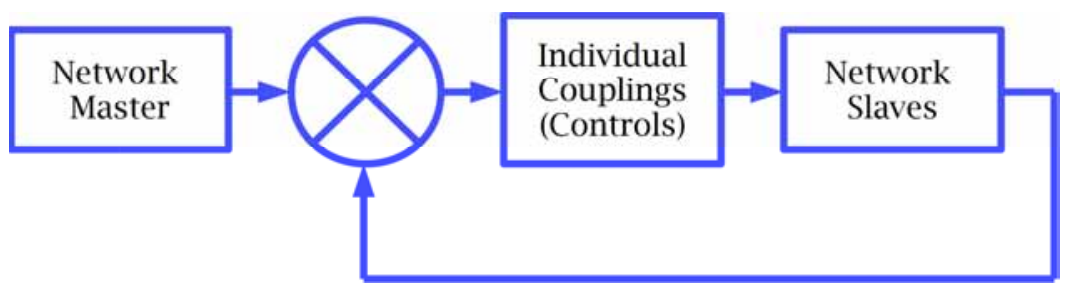

Fig. (1). Synchronization scheme. 


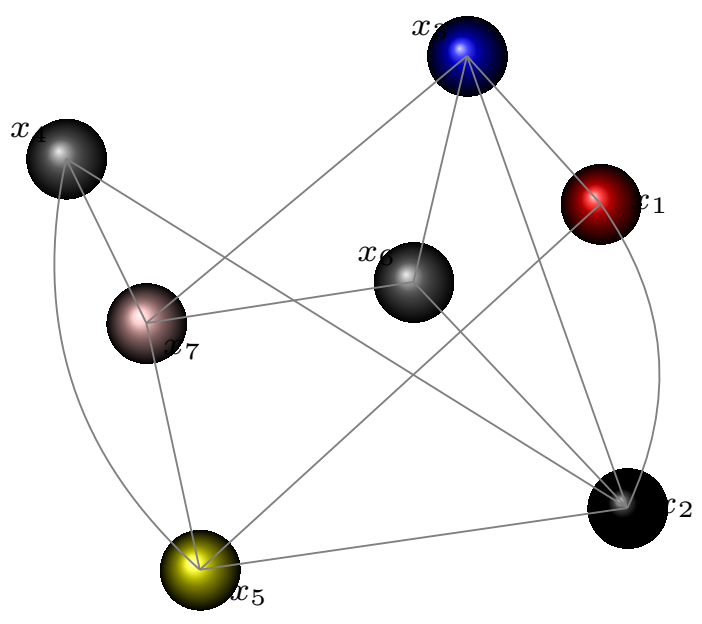

a

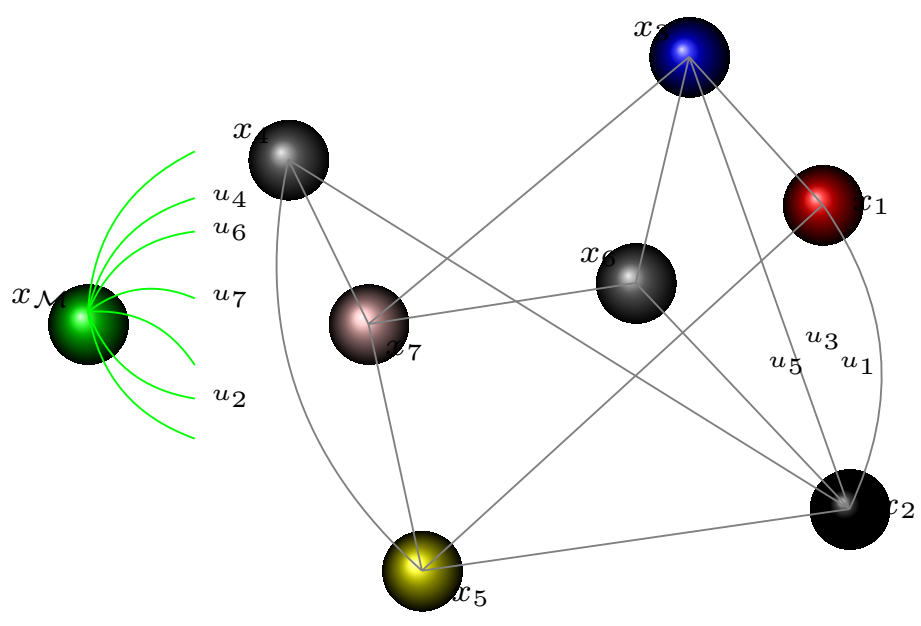

$\mathbf{E}$

Fig. (2). a) Fractional Complex Network, b) Master System $x_{\mathcal{M}}$ and the Slave systems $x_{i}$.

$\left|\arg \left(\operatorname{spec}\left(A_{i}+B_{i}\right)\right)\right|>\alpha \pi / 2$ and $\alpha \rho\left(A_{i}+B_{i}\right)>1, \quad$ for $k=1,2, \cdots, n$; finally we add a master system as in (10) to the network, see Fig. (2). Then we have that the interconnections defined by the equation

$$
\begin{aligned}
u_{i}= & A_{\mathcal{M}} x_{\mathcal{M}}+f_{\mathcal{M}}\left(x_{\mathcal{M}}\right)-A_{i} x_{\mathcal{M}}-B_{i}\left(x_{\mathcal{M}}-x_{i}\right)- \\
& \mathcal{F}_{i i}\left(x_{\mathcal{M}}-x_{i}\right)-\sum_{j=1}^{N} \mathcal{A}_{i j} x_{j}-\sum_{j=1}^{N} \mathcal{F}_{i j}\left(x_{j}\right)
\end{aligned}
$$

Ensure the practical synchronization of the complex network (11) with the master system (10)

Proof. Considering the error synchronization equation (12), and take its derivative of order $\alpha$

$$
\begin{aligned}
e_{i}^{(\alpha)}= & x_{\mathcal{M}}^{(\alpha)}-x_{i}^{(\alpha)}=A_{\mathcal{M}} x_{\mathcal{M}}+f_{\mathcal{M}}\left(x_{\mathcal{M}}\right)- \\
& \left(A_{i} x_{i}+\sum_{j=1}^{N} \mathcal{A}_{i j} x_{j}+\sum_{j=1}^{N} \mathcal{F}_{i j}\left(x_{j}\right)+u_{i}\right),
\end{aligned}
$$

replacing (13) in (14) we have

$e_{i}^{(\alpha)}=\left(A_{i}+B_{i}\right) e_{i}+\mathcal{F}_{i i}\left(e_{i}\right)$.

Now, due to our earlier supposition we have $\mathcal{F}_{i i}(0)=0$ and $\lim _{x \rightarrow \infty} \frac{\left\|\mathcal{F}_{i i}\left(x_{i}\right)\right\|}{\left\|x_{i}\right\|}=0$, we only need to choose $B_{i} \in \mathbb{R}^{n \times n}$ in such a way that $\left|\arg ?\left(\operatorname{spec}\left(A_{i}+B_{i}\right)\right)\right|>\alpha \pi / 2$ and $\alpha \rho\left(A_{i}+B_{i}\right)>1$, for $k=1,2, \cdots, n ;$; but we have already asked for such $B_{i}$ as hypothesis, thus by Theorem 2 we have that the origin of each of the fractional order differential nonlinear synchronization error systems of commensurate fractional order is stable, which gives us the practical synchronization of the entire network with the master system.

\subsection{Exact Synchronization of Fractional Complex Networks}

Now for the case of $1<\alpha<2$, we proceed in a similar way. Theorem 3 can be used to ensure the stability of a partially autonomous differential nonlinear system, which is said to be partially autonomous because the nonlinear part can be time-varying and Theorem 3 gives sufficient conditions for the asymptotic stability of the origin, i.e., now we will have

$\lim _{t \rightarrow \infty}\left\|e_{i}(t)\right\|=0$.

Now for this result we let the nonlinear parts of the systems, represented by the equations (8) to (11), be partially nonautonomous. Rewriting equation (9) for a system with a nonautonomous nonlinear part we have

$x_{i}^{(\alpha)}=A_{i} x_{i}+\sum_{j=1}^{N} \mathcal{A}_{i j} x_{j}+\sum_{j=1}^{N} \mathcal{F}_{i j}\left(t, x_{j}\right)$,

and rewriting equation (10)

$x_{\mathcal{M}}^{(\alpha)}=A_{\mathcal{M}} x_{\mathcal{M}}+f_{\mathcal{M}}\left(t, x_{\mathcal{M}}\right)$

with initial conditions

$\left.x_{i}^{(\alpha-r)}(t)\right|_{t=t_{0}}=x_{i_{r-1}}, \quad r=1,2$,

and

$\left.x_{\mathcal{M}}^{(\alpha-r)}(t)\right|_{t=t_{0}}=x_{\mathcal{M}_{r-1}}, \quad r=1,2$,

where $\mathcal{F}_{i i}\left(t, x_{j}\right):\left[t_{0}, \infty\right) \times \mathbb{R}^{n} \rightarrow \mathbb{R}^{n}$ is a continuous function in which $\mathcal{F}_{i i}(t, 0)=0$; moreover, $\mathcal{F}_{i i}\left(t, x_{j}\right)$ fulfills the Lipschitz condition with respect to $x_{j}$.

Theorem 5 Consider a complex fractional network with elements of the form (17) (11), where $\mathcal{A}_{i j} \in \mathbb{R}^{n \times n}$, $\mathcal{F}_{i i}(t, 0)=0$ and $\mathcal{F}_{i i}\left(t, x_{i}\right)$ satisfies $\lim _{x \rightarrow \infty} \frac{\left\|\mathcal{F}_{i i}\left(t, x_{i}\right)\right\|}{\left\|x_{i}\right\|}=0$, for $i, j \in\{1,2, \cdots, N\}$. Moreover, assume that there are $B_{1}, B_{2}, \cdots, B_{n} \in \mathbb{R}^{n \times n} \quad$ such that $A_{i}+B_{i}$ has $\left|\arg \left(\operatorname{spec}\left(A_{i}+B_{i}\right)\right)\right| \neq 0, \quad\left|\arg \left(\operatorname{spec}\left(A_{i}+B_{i}\right)\right)\right|>\alpha \pi / 2 \quad$ and $\alpha+1 /\left\|A_{i}+B_{i}\right\|<2$, for $k=1,2, \cdots, n$; finally we add a 
master system as in (18) to the network. Then we have that the interconnections defined by the equation

$u_{i}=A_{\mathcal{M}} x_{\mathcal{M}}+f_{\mathcal{M}}\left(t, x_{\mathcal{M}}\right)-A_{i} x_{\mathcal{M}}-B_{i}\left(x_{\mathcal{M}}-x_{i}\right)-\mathcal{F}_{i i}\left(t, x_{\mathcal{M}}-x_{i}\right)$

$-\sum_{j=1}^{N} \mathcal{A}_{i j} x_{j}-\sum_{j=1}^{N} \mathcal{F}_{i j}\left(t, x_{j}\right)$

ensures the exact synchronization of the complex network (17) with the master system (18).

Proof. Considering the error synchronization equation (12), and take its derivative of order $\alpha$

$$
\begin{aligned}
& e_{i}^{(\alpha)}=x_{\mathcal{M}}^{(\alpha)}-x_{i}^{(\alpha)}=A_{\mathcal{M}} x_{\mathcal{M}}+f_{\mathcal{M}}\left(t, x_{\mathcal{M}}\right) \\
& -\left(A_{i} x_{i}+\sum_{j=1}^{N} \mathcal{A}_{j j} x_{j}+\sum_{j=1}^{N} \mathcal{F}_{i j}\left(t, x_{j}\right)+u_{i}\right),
\end{aligned}
$$

replacing (21) in last equation

$e_{i}^{(\alpha)}=\left(A_{i}+B_{i}\right) e_{i}+\mathcal{F}_{i i}\left(t, e_{i}\right)$.

In a similar way that in the proof of Theorem 4, we already have asked for $\mathcal{F}_{i i}\left(t, e_{i}\right)$ to satisfy the second hypothesis from Theorem 3 , we only need to choose $B_{i} \in \mathbb{R}^{n \times n}$ in such a way that $A_{i}+B_{i}$ satisfies the first part of the hypothesis from Theorem 3 but we also have asked for $A_{i}+B_{i}$ to hold such conditions, and as a consequence we get the desired result.

\section{NUMERICAL EXAMPLE}

In this section we deal with a slave network whose components are fractional order differential systems: two Lorenz systems and one Chen system. The master will be a Lü system. The method introduced in [51] implies that the system has a unique solution. For the simulations we have taken the approximation of the fractional order operators presented in [52], based on [53]. First we present the individual systems of the network.

We take into account the modified fractional order Lorenz system [25], i. e.,

$x_{i}^{(\alpha)}(t)=A_{i} x_{i}+f_{i}\left(x_{i}\right)$

$=\left(\begin{array}{ccc}-a_{i} & a_{i} & 0 \\ b_{i} & -c_{i} & 0 \\ 0 & 0 & d_{i}\end{array}\right) x_{i}+\left(\begin{array}{c}0 \\ -x_{i 1} x_{i 3} \\ x_{i 1} x_{i 2}\end{array}\right)$ where $x_{i}=\left[\begin{array}{lll}x_{i 1} & x_{i 2} & x_{i 3}\end{array}\right]^{T}$. The case that $a_{i}=10$, $b_{i}=28, c_{i}=-8, d_{i}=8 / 3$ and $\alpha=0.8$ is shown in Fig. (3a).

The fractional order Lü system [35], i. e.,

$$
\begin{aligned}
& x_{\mathcal{M}}^{(\alpha)}(t)=A_{\mathcal{M}} x_{\mathcal{M}}+f_{i}\left(x_{\mathcal{M}}\right) \\
& =\left(\begin{array}{ccc}
-a_{\mathcal{M}} & a_{\mathcal{M}} & 0 \\
0 & c_{\mathcal{M}} & 0 \\
0 & 0 & -b_{\mathcal{M}}
\end{array}\right) x_{i}+\left(\begin{array}{c}
0 \\
-x_{\mathcal{M} 1} x_{\mathcal{M} 3} \\
x_{\mathcal{M} 1} x_{\mathcal{M} 2}
\end{array}\right)
\end{aligned}
$$

where $\quad x_{\mathcal{M}}=\left[\begin{array}{lll}x_{\mathcal{M} 1} & x_{\mathcal{M} 2} & x_{\mathcal{M} 3}\end{array}\right]^{T}$. The case that $a_{\mathcal{M}}=10, b_{\mathcal{M}}=3, c_{\mathcal{M}}=28$ and $\alpha=0.8$ is shown in Fig. (3b).

And the fractional order Chen system [30], i. e.,

$$
\begin{aligned}
& x_{i}^{(\alpha)}(t)=A_{i} x_{i}+f_{i}\left(x_{i}\right) \\
& =\left(\begin{array}{ccc}
-a_{i} & a_{i} & 0 \\
c_{i}-a_{i} & c_{i} & 0 \\
0 & 0 & -b_{i}
\end{array}\right) x_{i}+\left(\begin{array}{c}
0 \\
-x_{i 1} x_{i 3} \\
x_{i 1} x_{i 2}
\end{array}\right)
\end{aligned}
$$

where $x_{i}=\left[\begin{array}{lll}x_{i 1} & x_{i 2} & x_{i 3}\end{array}\right]^{T}$. See Fig. (3c) to observe the behavior of this system for $a_{i}=35, b_{i}=3, c_{i}=28$, $d_{i}=8 / 3$ and $\alpha=0.8$.

Let us take a complex network with, two Lorenz's systems for $i=1,2$, and a Chen's system for $i=3$, the bidirectional coupling scheme proposed in [18] is used; so we have that the elements of the network are of the form

$$
x_{i}^{(\alpha)}(t)=A_{i} x_{i}+f_{i}\left(x_{i}\right)+\sum_{j=1}^{3} H_{i j}\left(x_{j}\right)
$$

where

$$
H_{i j}\left(x_{j}\right)=\left(\begin{array}{ccc}
\sigma_{i j} d_{i 1} & 0 & 0 \\
0 & \sigma_{i j} d_{i 2} & 0 \\
0 & 0 & \sigma_{i j} d_{i 3}
\end{array}\right)\left(\begin{array}{c}
x_{j 1} \\
x_{j 2} \\
x_{j 3}
\end{array}\right), \sigma_{i j}=\left\{\begin{array}{c}
-2, \text { if } i=j \\
1, \text { if } i \neq j
\end{array}\right.
$$

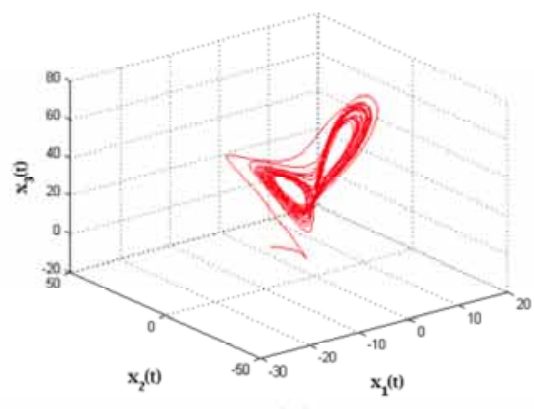

(a)

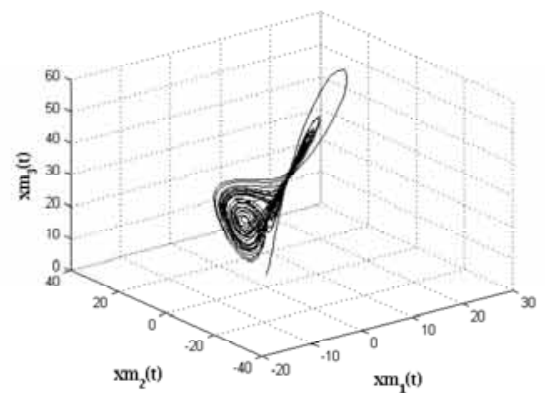

(b)

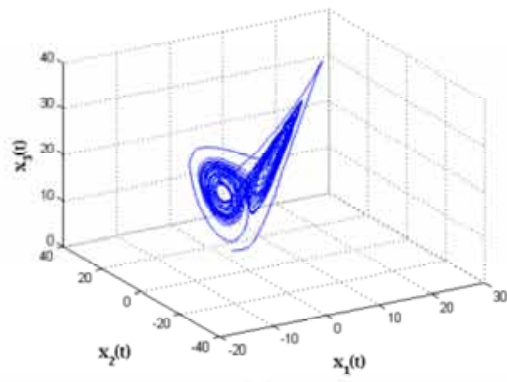

(c)

Fig. (3). Graphs of the fractional-order nonlinear systems of the network; (a) Lorenz system, (b) Lü system, (c) Chen system. 


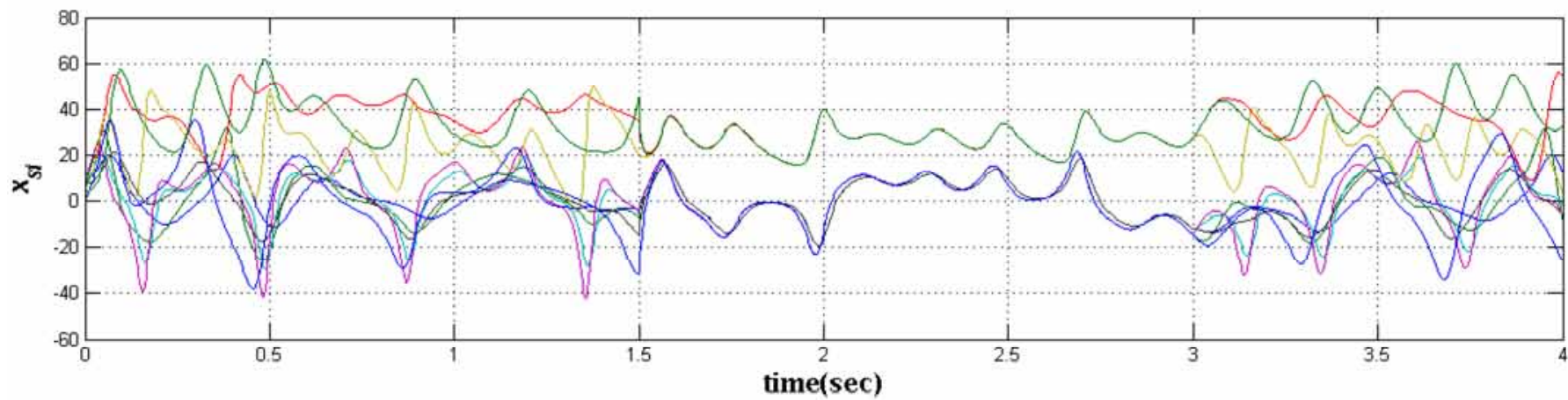

a)

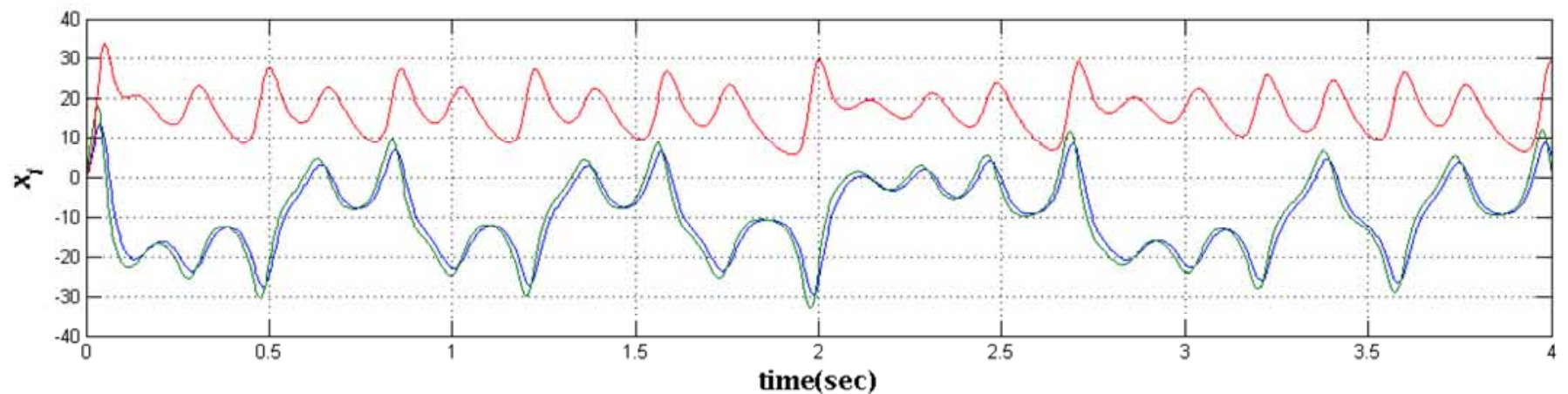

b)

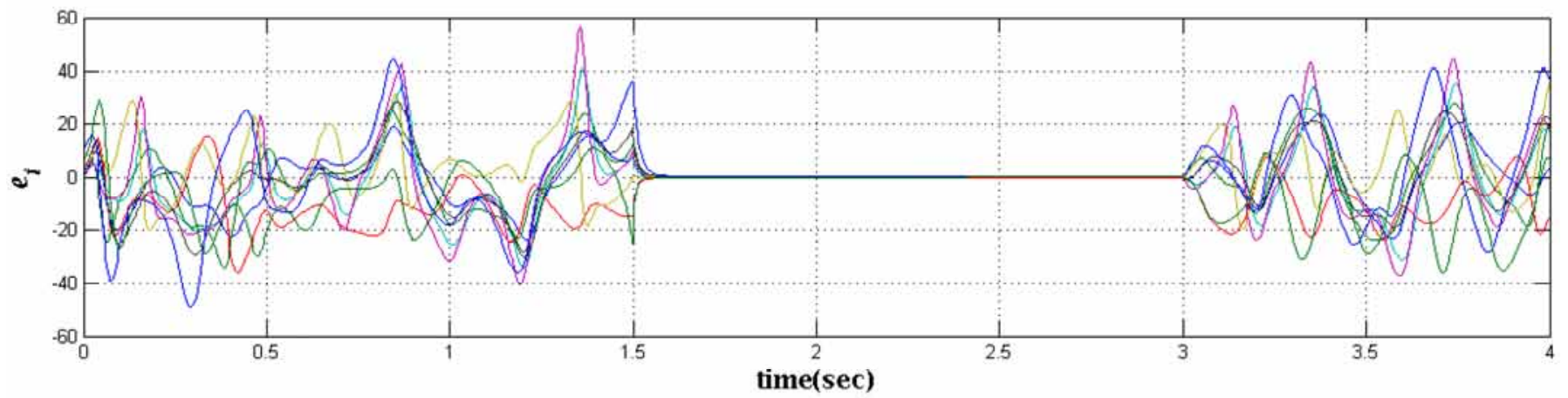

c)

Fig. (4). In the simulations we turn on the corresponding interconnections $u_{i}$ on $t=1.5 \mathrm{~s}$, and then we turned it off on $t=3 \mathrm{~s}$.

and we consider that the network can be separated in a linear plus a nonlinear part; in such case, the nonlinear part in each system is $\mathcal{F}_{11}=\mathcal{F}_{22}=\mathcal{F}_{33}$ and we have

$0 \leq \sqrt{\frac{x_{i 1}^{2} x_{i 3}^{2}+x_{i 1}^{2} x_{i 2}^{2}}{x_{i 1}^{2}+x_{i 2}^{2}+x_{i 3}^{2}}} \leq \sqrt{x_{i 3}^{2}+x_{i 2}^{2}}$,

and applying the limit when $\|x\| \rightarrow 0$ we have that hypothesis 2 from Theorem 2 holds. Now let us consider a Lü system as the master and construct the interconnections as in (13), thus having

$$
\begin{aligned}
u_{i}= & A_{\mathcal{M}} x_{\mathcal{M}}+f_{\mathcal{M}}\left(x_{\mathcal{M}}\right)-A_{i} x_{\mathcal{M}}-B_{i}\left(x_{\mathcal{M}}-x_{i}\right)- \\
& \mathcal{F}_{i i}\left(t, x_{\mathcal{M}}-x_{i}\right)-\sum_{j=1}^{3} \mathcal{A}_{i j} x_{j}-\mathcal{F}_{i i}\left(x_{i}\right)
\end{aligned}
$$

here $\mathcal{A}_{i j}=H_{i j}$ wich implies that the equations of the synchronization error will be of the kind of (15). With the election of

$$
B_{1}=B_{2}=B_{3}=\left(\begin{array}{ccc}
-50 & 0 & 0 \\
0 & -50 & 0 \\
0 & 0 & -50
\end{array}\right)
$$

the first condition from Theorem 2 holds, specifically, the term $A_{1}+B_{1}$ has the eigenvalues $-70,-32,-52.66$ and $\alpha \rho(A)=56$, the graphs are shown in Fig. (4)

We take the parameters of $H_{i j}$ as $d_{11}=-1, d_{12}=2$, $d_{13}=-3, \quad d_{21}=4, \quad d_{22}=2, d_{23}=-3, d_{31}=100 / 29$, 
$d_{32}=-1$ and $d_{33}=17 / 48$, where the selection of these values was arbitrary.

Before the activation of the interconnection we observe the behavior in each of the states of the complex network and when activated, the corresponding states of the network synchronize with the corresponding master system. We can also observe that the error approximates zero when the interconnection is turned on (see Fig. 4).

\section{CONCLUSIONS}

In this work we use a coupling scheme to achieve the fractional complex network synchronization in a MasterSlave scheme, where all the elements of the network are fractional order dynamical systems. As a specific difference with other works, the results used herein allow us to let the dynamical error be nonlinear. The respective coupling $u_{i}$ is nonlinear and does not cancel completely the nonlinear part of each system, thus the practical synchronization and the exact synchronization are respectively guaranteed, despite this nonlinearities.

In particular, the results obtained are applicable when we have different fractional linear systems, when we have fractional linear systems of the same type, or when we are working with a linearization of the original nonlinear systems or we consider a system-to-system synchronization scheme, because it turns out that these schemes are particular conditions of the family of complex networks treated herein. For linear systems with their orders lying in $(1,2)$ we only need to use Theorem 3.3 from [49].

It is important to notice that the hypothesis on each node is applicable also to other kinds of fractional dynamical systems [32, 54], and not only to that used in the example presented here.

\section{CONFLICT OF INTEREST}

The authors confirm that this article content has no conflict of interest.

\section{ACKNOWLEDGEMENTS}

Declared none.

\section{REFERENCES}

[1] A. Pikovsky, M. Rosenblum, and J. Kurths, Synchronization: A universal concept in nonlinear Sciences. University Press: Cambridge, 2001.

[2] E. Mosekilde, Y. Maistrenko, and D. Postnov. Chaos Synchronization: Applications to Living Systems. World Scientific Publishing Co. Pte. Ltd., 2002.

[3] R. Albert and A. Barabasi, "Statistical mechanics of complex networks", Rev. Modern Phys., vol. 74, pp. 47-91, 2004,

[4] S. N. Dorogovstev and J. F. F. Mendes, "Evolution of networks", Adv. Phys., vol. 51, no. 4, pp. 1079-1157, 2002

[5] X. Li and G. Chen, "A local-world evolving network model", Phys. A., vol. 328, pp. 274-286, 2003.

[6] M. Barahona and L. M. Pecora, "Synchronization in small-world systems", Phys. Rev. Lett., vol. 89(5), p. 054101, 2002.

[7] H. Hong and M. Y. Choi, "Synchronization on small-world networks", Phys. Rev. E., vol. 65, p. 026139, 2002.

[8] J. Lu., X. Yu, G. Chen, and D. Cheng, "Characterizing the synchronizability of small-world dynamical networks", IEEE Trans. Circ. Syst. I., vol. 51, pp. 787-796, 2004.
[9] L. F. Lago-Fernandez, R. Huerta, F. Corbacho, and J. A. Siguenza, "Fast response and temporal coherent oscillations in small-world networks", Phys. Rev. Lett., vol. 84, no. 12, pp. 2758-2761, 2000.

[10] W. Lu and T. Chen, "Synchronization of coupled connected neural networks with delays", IEEE Trans. Circ, Syst. I., vol. 51, pp. 2491-2503, 2004.

[11] I. Podlubny, Fractional Differential Equations. $9^{\text {th }}$ ed. Academic Press: California, 1999.

[12] R. Hilfer, Applications of fractional Calculus in Physics. World Scientific, River Edge: New Jersey, 2000.

[13] R. L. Bagley and R. A. Calico, "Fractional order state equations for the control of viscoelastically damped structures", J. Guid. Control Dyn., vol. 14, pp. 304-311, 1991.

[14] G. Peng and Y. Jiang, "Generalized projective synchronization of a class of fractional-order chaotic systems via a scalar transmitted signal", Phys. Lett. A., vol. 372, pp. 3963-3970, 2008.

[15] G. Peng, "Synchronization of fractional order chaotic systems", Phys. Lett. A., vol. 363, pp. 426-432, 2007.

[16] J.-W. Wang and Y.-B. Zhang, "Synchronization in coupled nonidentical incommensurate fractional-order systems," Phys. Lett. A., vol. 374, pp. 202-207, 2009.

[17] W. Xingyuan and H. Yijie, "Projective synchronization of fractional order chaotic system based on linear separation", Phys. Lett. A., vol. 372, pp. 435-441, 2008.

[18] Y. Yu, H.-X. Li, and Y. Su, "The synchronization of three chaotic fractional-order lorenz systems with bidirectional coupling", $J$. Phys. Conf. Ser., vol. 96, no. 1, p. 012113, 2008.

[19] A. Matouk, "Stability conditions, hyperchaos and control in a novel fractional order hypechaotic system", Phys. Lett. A., vol. 373, pp. 2166-2173, 2009.

[20] Y. Zheng, Y. Nian, and D. Wang, "Controlling fractional order chaotic systems based on Takagi-Sugeno fuzzy model and adaptive adjustment mechanics", Phys. Lett. A., vol. 375, pp. 125-129, 2010.

[21] M. S. Tavazoei and M. Haeri, "Chaos control via a simple fractional-order controller," Phys. Lett. A., vol. 372, pp. 798-807, 2008.

[22] S. Momani and S. Hadid, "Lyapunov stability solutions of fractional integro-differential equations", IJMMS., pp. 2503-2507, 2003.

[23] E. Ahmed, A. El-Sayed, and Hala A.A. El-Saka, "On some RouthHurwitz conditions for fractional order differential equations and their applications in Lorenz, Rössler, Chua and Chen systems", Phys. Lett. A., vol. 358, pp. 1-4, 2010.

[24] D. Matignon, Ed. "Stability results for fractional differential equations with applications to control processing," In: Proc. IMACS, IEEE-SMC., pp. 963-968, 1996.

[25] X.-J. Wen, Z.-M. Wu, and J.-G. Lu, "Stability analysis of a class of nonlinear fractional-order systems”, IEEE Trans. Circ. Syst-II., vol. 55, pp. 1178-1182, 2008.

[26] J. Wang and Y. Zhang, "Network synchronization in a population of star-coupled fractional nonlinear oscillators", Phys. Lett. A., vol. 374, pp. 1464-1468, 2010.

[27] D. Kusnezov, A. Bulgac, and G. D. Dang, "Quantum levy processes and fractional kinetics", Phys. Rev. Lett., vol. 82, pp. 1136-1139, 1999.

[28] Y. Tang and J.-A. Fang, "Synchronization of n-coupled fractionalorder chaotic systems with ring connection", Commun. Nonlinear Sci., vol. 15, no. 2, pp. 401-412, 2010.

[29] A. Kiani-B, K. Fallahi, N. Pariz, and H. Leung, "A chaotic secure communication scheme using fractional chaotic systems based on an extended fractional Kalman filter", Commun. Nonlinear Sci., vol. 14 , no. 3 , pp. 863-879, 2009.

[30] C. Li and G. Chen, "Chaos in the fractional order Chen system and its control", Chaos. Soliton. Fract., vol. 22, pp. 549-554, 2004.

[31] I. Grigorenko and E. Grigorenko, "Chaotic dynamics of the fractional Lorenz system”, Phys. Lett. A., vol. 91, no. 3, pp. 034 101:1-4, 2003.

[32] T. T. Hartley, C. F. Lorenzo, and H. K. Qammer, "Chaos in a fractional order Chua 's system”, IEEE. T Circ-I., vol. 42, pp. 485490, 1995.

[33] G. Peng, Y. Jiang, and F. Chen, "Generalized proyective synchronization of fractional order chaotic systems", Phys. A., vol. 387, pp. 3738-3746, 2009.

[34] X.-Y. Wang and J.-M. Song, "Synchronization of the fractional order hyperchaos Lorenz systems with activation feedback 
control", Commun. Nonlinear Sci. Numer. Simulat., vol. 14, pp. 3351-3357, 2009

[35] J. G. Lu, "Chaotic dynamics of the fractional-order Lü systems and its synchonization", Phys. Lett. A., vol. 354, pp. 305-311, 2006.

[36] Y. Tang and J. an Fang, "Syncronization of n-coupled fractionalorder chaotic systems with ring connection", Commun. Nonlinear Sci. Numer. Simulat., vol. 15, pp. 401-412, 2010.

[37] A. E. Matouk, "Chaos, feedback control and synchronization of a fractional-order modified autonomous van der Pol-Duffing circuit", Commun. Nonlinear Sci. Numer. Simulat., vol. 16, pp. 975-986, 2011.

[38] M. Shahiri, R. Ghaderi, A. R. N., S. H-Hosseinnia, and S. Momani, "Chaotic fractional-order Coullet system: Synchronization and control approach", Commun. Nonlinear Sci. Numer. Simulat., vol. 15, pp. 665-674, 2010.

[39] S. Bhalekar and V. Daftardar-Gejji, "Synchronization of different fractional order chaotic systems using active contol", Сотmun. Nonlinear Sci. Numer. Simulat., vol. 15, pp. 3536-3546, 2010.

[40] H. Taghvafard and G. H. Erjaee, "Phase and anti-phase synchronization of fractional order chaotic systems via active control", Commun. Nonlinear Sci. Numer. Simulat., vol. 16, pp. 4079-4088, 2011.

[41] K. S. Miller and B. Ross, An Introduction to the Fractional Calculus and Fractional Differential Equations. John Wiley \& Sons: New York, 1993.

[42] I. Podlubny, "Geometric and physical interpretation of fractional integration and fractional differentiation", Fract. Calculus Appl. Anal., vol. 5, pp. 367-386, 2002.

[43] M. Ortigueira and F. Coito, "Initial conditions: what are we talking about?" Third IFAC Workshop on fractional differentiation, pp. 0507, 2008.

[44] J. Sabatier, M. Merveillaut, R. Malti, and A. Oustaloup, "How to impose physical coherent initial conditions to a fractional system?",
Commun. Nonlinear Sci. Numer. Simulat., vol. 91, pp. 1318-1326, 2010.

[45] J. Trigeassou and N. Maamri, "Initial conditions and initialization of linear fractional differential equations", Signal Process., vol. 91 pp. 427-436, 2011.

[46] T. J. Claude, M. Nezha, and O. Alain, "Initialization of RiemannLiouville and Caputo fractional derivatives", In: Proceedings of the ASME, Bordeaux: France, 2011.

[47] N. Achar, F. Lorenzo, and T. Hartley, "Initialization and the Caputo fractional derivative", NASA John H. Glenn Research Center at Lewis Field, vol. report, 2003.

[48] A. A. Kilbas, H. M. Srivastava, and J. J. Trujillo, Theory and Applications of Fractional Differential Equations, $1^{\text {st }}$ ed. Elsevier: 2006.

[49] F. Zhang and C. Li, "Stability analysis of fractional di_erential systems with order lying in (1,2)", Adv. Differ. Equ., p. 17, 2011.

[50] J. P. García-Sandoval, R. Femat, and V. González-Álvarez, "On generalized synchronization of different-order chaotic systems: a submanifold approach", Phys. A: Math. Theor., vol. 42, p. 15, 2009.

[51] Y. Yu, H.-X. Li, S. Wang, and J. Yu, "Dynamic analysis of a fractional-order Lorenz chaotic system," Chaos, Solitons Fractals, vol. 42, pp. 1181-1189, 2009.

[52] T. T. Hartley, C. F. Lorenzo, and H. K. Kammer, "Chaos in a fractional order Chua's system," IEEE Trans. CAS-I., vol. 42, pp. 485-490, 1995 .

[53] A. Charef, H. H. Sun, Y. Y. Tsao, and B. Onaral, "Fractal system as represented by singularity function," IEEE Trans. Automatic. Control., vol. 37, pp. 1465-1470, 1992.

[54] Z. Xiao-Dan, Z. Pin-Dong, and L. Ai-Hua, "Construction of a new fractional chaotic system and generalized synchronization" Commun. Theor. Phys., vol. 53, pp. 1105-1110, 2010.

(C) Martínez-Martínez et al.; Licensee Bentham Open.

This is an open access article licensed under the terms of the Creative Commons Attribution Non-Commercial License (http://creativecommons.org/licenses/by-nc/3.0/) which permits unrestricted, non-commercial use, distribution and reproduction in any medium, provided the work is properly cited. 